\title{
Hypertonic Saline in Acute Bronchiolitis: Is It Worth the Salt?
}

\author{
M JAYASHREE \\ Department of Pediatrics, Advanced Pediatrics Centre, \\ Postgraduate Institute of Medical Education and Research, Chandigarh. \\ mjshree@hotmail.com
}

A cute viral bronchiolitis is the commonest lower respiratory tract infection seen during infancy [1]. Despite its frequency, there is no single widely-practised evidence based treatment approach [2]. Bronchodilators still remain the most prescribed, albeit without sound evidence for favorable clinical outcome [3]. Supportive treatment, ensuring adequate oxygen exchange and fluid intake, still remains the standard of care [4].

Peribronchial inflammation, airway edema, mucus plugging and necrosis, and desquamation of ciliated epithelial cells are the predominant pathological processes implicated in acute bronchiolitis [4]. Theoretically, any therapeutic modality which can improve clearance of airway secretions and minimize edema should be beneficial. Four such modalities that have been studied targeting the above are inhaled epinephrine, recombinant deoxyribonuclease (rhDNase), chest physiotherapy and hypertonic saline [1]. Of these, hypertonic saline has recently shown some promising results, the basic premise for its use stemming from extrapolation of its benefits seen in asthma, bronchiectasis, cystic fibrosis and sinonasal disease [4]. It has been postulated that saline hydrates airway surface liquid, improves impaired mucociliary clearance and aids water absorption from the mucosa, thereby reducing airway edema [1].

A recently published Cochrane Systematic Review of hypertonic vs. $0.9 \%$ saline in mild to moderate acute bronchiolitis found significantly shorter length of hospital stay as well as a lower clinical severity score in the former as compared to the latter [4]. The effect sizes of treatment with $3 \%$ saline reported by the four independent studies in the review were similar despite differences in inhalation mixture and delivery intervals across the studies. Hypertonic saline achieved a reduction in length of hospital stay of 1.16 days $(24.1 \%)$ compared to the normal saline arm.
The study by Sharma, et al. [5] in this issue, though very similar in methodology but larger in numbers as compared to the above trials has reported findings to the contrary; they failed to show a significant difference in the clinical severity scores as well as length of hospital stay between $3 \%$ and $0.9 \%$ saline groups. In fact a closer look at the results of the studies reporting improvement with $3 \%$ saline reveals that the magnitude of improvement differed on different treatment days varying from $15.7 \%$ on day 1 to $29.4 \%$ on day 3 . Further, studies conducted among outpatients failed to replicate the advantage seen in inpatients, once again raising the question about the utility of $3 \%$ saline in the early period of the disease [4]. It is a moot point therefore whether the positive results seen with hypertonic saline in hospitalized children in previous trials can be ascribed to the intervention or coincident natural recovery.

Several studies have looked into the concentration and volume of saline used, vis-a-vis clinical outcomes. It has been shown that the change in airway surface liquid depth is a direct result of total mass of sodium chloride added to the airway surface and can be altered by both increasing the concentration and lowering the volume or vice versa [4]. Hypertonic saline in concentrations of $3 \%$, $7 \%$ and $12 \%$ have shown promising results in a dose response pattern in patients with cystic fibrosis [6]. Similar concentration-dependent improvement was noted by Ansari, et al. [6] at 48 hours of treatment in a small sample outpatient study of acute bronchiolitis. Sood, et al. [7] reported increased rates of mucociliary clearance in normal subjects with increasing volume of airway surface liquid. This was substantiated by Anil, et al. [4], who reported improvement in clinical scores with high volume of $0.9 \%$ saline. Volume-related improvements; however, have been mostly seen in mild cases and cannot be extrapolated to moderate or severe disease wherein use of large volumes of normal saline may be risky. Most of the studies have used saline in volumes varying from 2-4 $\mathrm{mL}[4]$. 


\title{
EDITORIALS
}

The ideal frequency of nebulized $3 \%$ saline is unclear, though most studies have found multiple daily doses for several days to be effective as opposed to repeated inhalations over a short period [4]. This possibly can serve as a major advantage over inhaled epinephrine where repeated use can be limited by tachycardia and/or rebound mucosal edema. Ralston, et al. [8] in their study found a low rate of adverse events when hypertonic saline was used without adjunctive bronchodilators. Such reports; however, are far and few as most studies on hypertonic saline in bronchiolitis including the current one [5] have combined it with some bronchodilator to counter the theoretical risk of precipitating bronchospasm.

The airway clearance properties, safety profile, feasibility of repeated administrations, and costeffectiveness of hypertonic saline make it an ideal intervention in a setting where most of the studied interventions have failed. Though the current body of evidence seems to favor the routine use of nebulized 3\% saline in hospitalized infants with mild to moderate acute bronchiolitis, the study by Sharma, et al. [5] has provided more food for thought. The questions that need to be addressed before it becomes standard of care for acute bronchiolitis are its generalizability in every set up, its utility in outpatients and severe disease, the need for adjunctive bronchodilator therapy, and the optimal concentration and dosing intervals.

Funding: Nil; Competing interests: None stated.

\section{REFERENCES}

1. Bialy L, Foisy M, Smith M, Fernandes RM. The Cochrane library and the treatment of bronchiolitis in children: An overview of reviews. Evid-Based Child Health. 2011:6; 258-275.

2. Zhang L, Mendoza-Sassi RA, Wainwright C, Klassen TP. Nebulised hypertonic saline solution for acute bronchiolitis in infants. Cochrane Database Syst Rev. 2008;4:CD006458.

3. Anil AB, Anil M, Saglam AB, Cetin N, Bal A, Aksu N. High volume normal saline alone is as effective as nebulized salbutamol-normal saline, epinephrine- normal saline, and $3 \%$ saline in mild bronchiolitis. Pediatr Pulmonol. 2010; 45:41-7.

4. De Brasi D, Pannuti F, Antonelli F, de Seta F, Siani P, de Seta L. Therapeutic approach to bronchiolitis: why pediatricians continue to overprescribe drugs? Ital J Pediatr. 2010;36:67-74.

5. Sharma BS, Gupta MK, Rafik SP. Hypertonic (3\%) saline for acute viral bronchiolitis: A randomized trial. Indian Pediatr. 2013;50:743-7.

6. Khalid AA, Sakran M, Bruce L, Davidson, Sayyed RE, Mahjoub $\mathrm{H}$, et al. Nebulized $5 \%$ or $3 \%$ hypertonic or $0.9 \%$ saline for treating acute bronchiolitis in infants. $\mathrm{J}$ Pediatr. 2010; 157:630-4.

7. Sood N, Bennett WD, Zeman K, Brown J, Foy C, Boucher $\mathrm{RC}$, et al. Increasing concentrations of inhaled saline with or without amiloride: effect on mucociliary clearance in normal subjects. Am J Respir Crit Care Med. 2003;167:158-63.

8. Ralston S, Hill V, Martinez M. Nebulized hypertonic saline without adjunctive bronchodilators for children with bronchiolitis. Pediatrics. 2010; 126; e 520-5.

\section{Position of Central Venous Catheters in Children}

\author{
Banani Poddar \\ Department of Critical Care Medicine, Sanjay Gandhi Postgraduate Institute of Medical Sciences, Lucknow, India. \\ bananip@sgpgi.ac.in
}

$\mathrm{T}$ he correct positioning of central venous catheters in pediatric patients is a task not easily achieved and is complicated by the fact that the patients differ considerably in size depending on their age, anthropometry and nutritional status. The study by Witthayapraphakorn, et al. [1] adds to the scarce literature on this topic.

While there is no clear consensus regarding the position of the tip of central venous catheters (CVC) in the superior vena cava (SVC) [2], the right atrium (RA) should definitely be avoided due to the risk of vascular/ cardiac perforation. The SVC at the level of the carina is preferred by some authors, while others prefer the junction of the right atrium with the SVC (RA-SVC junction). Autopsy studies on infants have shown that the carina is almost always located above the pericardial reflection on the SVC [3]. Thus using the carina as a landmark for placement of the tip of the CVC reliably excludes placement in the RA. The carina is relatively easy to identify either by the anatomical landmark method or by radiological evaluation.

Studies in children to guide the positioning of CVC 